\title{
Hybrid Approach of Hyperthermia and Controlled Drug Delivery in Cancer: Image Guided Cancer Theranostic Monitoring in Tumor Management
}

\author{
Rakesh Sharma ${ }^{1,2 *}$ \\ 1Innovations and Solutions Inc, Florida State University Research Foundation, USA
}

2Department of Medicine, Columbia University, USA

Submission: March 08, 2018; Published: June 13, 2018

"Correspondence Address: Rakesh Sharma, Innovations and Solutions Inc., Florida State University Research Foundation, Tallahassee, FL, Department of Medicine, Columbia University, New York, 10032, USA, Tel: 011-91-9548336632; Email: rksz2009@gmail.com

\begin{abstract}
Nonpathogenic bacteria coats filled with complex metal nanoparticles form multifunctional magnetic nanoparticles (MNPs) for targeting cancer tumors. The encapsulated metal nanoparticles along with anticancer drugs inside polymer or bacterial coats become physiologically tumor sensitive due to hysteresis and pH sensitivity and drug active covalent bonds. Drug-bearing bacterial and polymer nanoshells open and close at specific $\mathrm{pH}$ to release anticancer drugs in controlled manner at the tumor sites. Simultaneously, metal core generates multimodal images. Such combined effect of chemotherapy and tumor sensitive nanodrugs offers hybrid approach of an effective and minimal invasive protocol of tumor management. Delivery of nanoshells filled with drug and nanometal core at tumor targets, offers theranostic monitoring of cancer prevention. Presence of metal in the core of nanoshells also serves as a non-invasive imaging contrast agent for therapeutic and diagnostic monitoring to evaluate the success of cancer prevention in real time. Preliminary opinion reported in Nature Precedings, hdl:10101/ npre.2008.2190.1 suggested significant tumor relapse or reduced dose dependent neoplastic progress of tumor as possible mode of tumor management by real-time tumor imaging.to prolong the life. Nanoparticles made of drug conjugates and complexes, dendrimers, vesicles, micelles, core-shell particles, microbubbles, and carbon nanotubes also function as smart imaging contrast to visually monitor the tumor tissue behavior and functions
\end{abstract}

Keywords: Theranostic monitoring; Hyperthermia; Tumor; MRI; Nanoparticles; Salmonella nanoshells

\section{Introduction}

The superparamagnetic nanoparticles can target hypoxic tumor specific cells sparing the normal cells. Nanoparticles made of bacterial envelope, dendrimers, vesicles, micelles, core-shell particles, microbubbles, and carbon nanotubes filled with sufficient anticancer drugs or complexes may be delivered to achieve optimal drug chemosensitive effects at the tumor locations without any systemic side effects. However, the chemotherapeutic success of such nanodrugs depends on the diagnosis, targeted delivery, and monitoring the tumor biological and physiological changes visible as multimodal images of tumor territories. Such approach is called 'theranostic monitoring' of tumor targeting. These nanodrugs offer passive and active targeting, environmentally responsive controlled drug release, molecular imaging, and other therapeutic functions into a single platform. Still, challenges are poor image sensitivity, accuracy of targeting biomarkers, innate toxicity of the nanoparticle components, formulation instability, high production costs, and control of controlled drug release.
At high temperatures, tumor cells starve and loose viability due to hysteresis hyperthermic effect. Reduced viability of the cancerous cells increases sensitivity to chemotherapy and/or radiation at high tumor tissue temperatures up to $42-46^{\circ} \mathrm{C}$ [15]. Cancer cells are easily destroyed at $43^{\circ} \mathrm{C}$ while normal cells survive up to $46^{\circ} \mathrm{C}$. Different methods using hot water, capacitive heating, and inductive heating of malignant cells are modalities of hyperthermia [6-9]. Such hyperthermia devices are designed to heat malignant cells sparing the surrounding healthy normal tissues and cells [10-12]. However, therapeutic efficacy of hyperthermia is demonstrated in animal models and its use in human is still in infancy [13].

Iron oxide particles have been used as contrast agents because the iron particles shorten the effective transverse relaxation time (T2) of tissues that take up these particles. Gadolinium diethyltriamine pentaacetic acid (Gd-DTPA) has been also used as a contrast agent that primarily shortens longitudinal relaxation time (T1) resulting in intensity enhancement. Now different 


\section{Cancer Therapy \& Oncology International Journal}

metallic magnetic nanoparticles have emerged as smart functional theranostic agents at different field strengths and frequencies for encapsulation and controlled delivery of the nanoparticles at tumor targets [14-24]. In fact, magnetic particles are dispersed first throughout the target tissue and then AC magnetic field of appropriate strength and frequency is applied to heat the particles up to $43^{\circ} \mathrm{C}$. At this temperature, slowly cancerous cells are destroyed if tumor temperature is maintained for at least $30 \mathrm{~min}$. Here serious drawback is coincidental heating of the surrounding normal tissues. As a result, use of sole hyperthermia devices is restricted and unacceptable. Hybrid chemotherapy combined with hyperthermia serves as alternative by nonvirulent bacterial coats filled with drugs and metallic complex as effective nanoparticles in cancer theranosis or prevention.

Hybrid Approach of Hyperthermia and Controlled Nanosphere Drug Delivery Method

The concept of magnetic hyperthermia induced tumor killing is based on the principle that magnetic particles inside tumor cells generate temperature up to $43^{\circ} \mathrm{C}$ by hysteresis loss when exposed in a high frequency $\sim 1 \mathrm{MHz}$ magnetic field [25]. In fact, hyperactive malignant cells take up MNPs nine times more than normal cells do [26]. So, malignant cells get heat up to $43^{\circ} \mathrm{C}$ or more than the normal cells at below $42^{\circ} \mathrm{C}$. The Curie temperature of the magnetic nanoparticle may be between about $40^{\circ} \mathrm{C}$ and about $44^{\circ} \mathrm{C}$. Due to high vasculature in the cancer tissues the heat dissipation is much slower. Hence, the temperature in the tumor remains higher than in the surrounding normal tissues as antitumoral effect in areas with more concentrated heat.

Magnetic hyperthermia is achieved by micro- and nano-scale injectable colloidal dispersion of magnetic particles by three strategies: arterial immobilization hyperthermia (AEH), direct injection hyperthermia (DIH), and intracellular hyperthermia (IH) [27]. However, these three approaches lack the capacity to assess the loading and distribution of particles at the tumor site, continuous monitoring of the immobilization, and treatment progress. Image-guided chemotherapy evaluation and hyperthermia approach is other tumor treatment monitoring (Theranostic Monitoring) option using multifunctional magnetic nanoparticles. It has been shown as safe, effective multimodal approach with excellent tumor heating ability. Magnetic particles are used as imaging contrast agents and drug delivery agents in theranosis $[28,29]$. Currently used diagnostic molecular imaging modalities include MRI, CT, US, optical imaging (bioluminescence and fluorescence), single photon emission computed tomography (SPECT) and positron emission tomography (PET).

Hyperthermia therapy with nanoparticle assisted chemotherapy is used in cancers, cardiac arrhythmias treatment and heat sensitive gene therapy by monitoring temperature variations [30,31]. MRI based hyperthermia and thermometry was reviewed by author. MRI thermometry detects the temperature variation by measuring small changes in the proton resonant frequency [32], longitudinal relaxation time [33] or apparent diffusion coefficient [34]. These hyperthermia techniques suffer from poor temperature sensitivity and influenced by the local motion and magnetic susceptibility variations [35].

Liposome-encapsulated gadolinium chelate nanoparticles were reported sensitive to MRI phase changes [36]. The local tissue temperature variations during the phase transition can reconstruct temperature distribution map of different deep-seated tissue locations [36]. The magnetic moments and temperature dependent characteristics of MNPs define their image contrast properties during hyperthermia therapy of cancer tissues. Different magnetic moments of the tissue and MNPs showed additional contrast in magnetic resonance imaging [32]. Steep variation in magnetic moments of MNPs with temperature rise may monitor the local tissue temperatures [32]. However, liposomes may be rejected by tumor tissues. So, nonvirulent bacterial envelops are smart option as controlled drug release nanoshells. Genetically modified strains of Salmonella typhimurium bacteria accumulate at tumor sites when injected in tumor-bearing mice and clear rapidly from blood in normal mice. The innovative delivery system makes use of genetically modified strains of bacteria, which includes genetically stable attenuated virulence (deletion of purl gene), reduction of septic shock potential (deletion of msbB gene) and antibiotic susceptibility.

\section{Nonvirulent Bacterial Nanoshells in Nanomedicine}

Nonpathogenic bacteria strains are prepared as 'empty bag' nanoshells by genetic modification [36]. Please see the Appendix 1. These bacteria exhibit the natural affinity with tumors to target these tumors. Author reviewed multifunctional nanoparticles in the form of $\mathrm{ZnGdxFe}(2-\mathrm{x}) \mathrm{O}_{4}$ as samples of Gd-substituted $\mathrm{Zn}$ Ferrite nanoparticles synthesized using chemical coprecipitation method by adding $\mathrm{NaOH}$ solution to different metal salts (e.g., $\mathrm{FeCl}_{3}, \mathrm{Fe}_{2} \mathrm{SO}_{4}, \mathrm{ZnSO}_{4}, \mathrm{GdCl}_{3}$ ) [32]. After vigorous stirring and filtration, newly synthesized particles were washed and then allowed to air dry at room temperature to make them ready for bacterial nanoshells [37].

In following, results given in a feasibility study (Nature Precedings hdl:10101/npre.2008.2190.1) are critically analyzed useful for possible new antitumor drugs in tumor management plan.

\section{Characterization of Avirulent Bacterial Nanoshells} filled with $\mathrm{ZnGd-ferrite}$

Inside nanoshells, Gd-substituted Zn Ferrite particles and $\mathrm{Zn}$-Ferrite particles added with Gd in small amounts may increase temperature called Curie temperature (Tc) as well as the pyromagnetic co-efficient of the nanoparticles. Critical analysis: At high Curie temperatures, bacterial nanoshells fail to maintain viable membrane pores due to temperature sensitive pore protein ionophores (Figure 1). The XRD pattern confirms the formation of single-phase structure as shown in Figure 2. Critical Comment: $\mathrm{X}$-Ray diffraction pattern shows multi-phase structure. 


\section{Cancer Therapy \& Oncology International Journal}
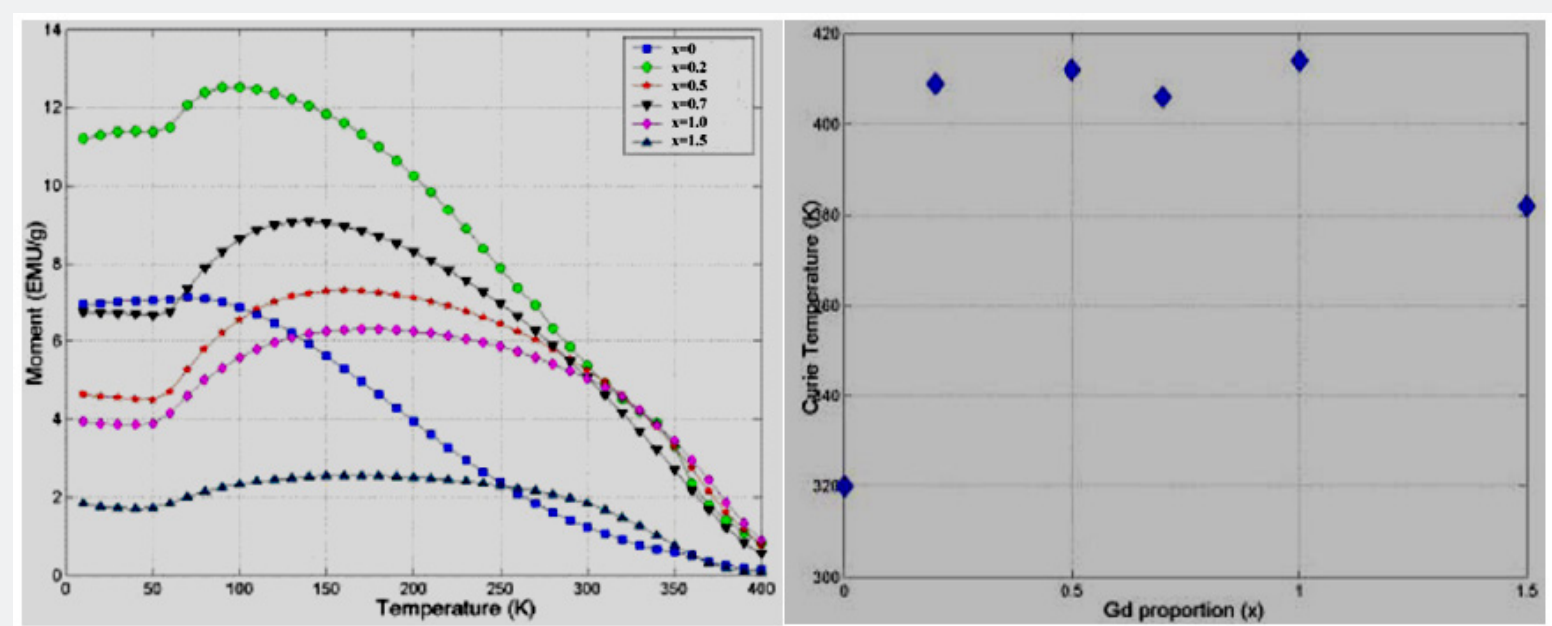

Figure 1: A. The magnetic moment of particles as a function of temperature.

B. The variation of their Tc as a function of $x$.



Figure 2: X-ray diffraction pattern of single-phase structure.
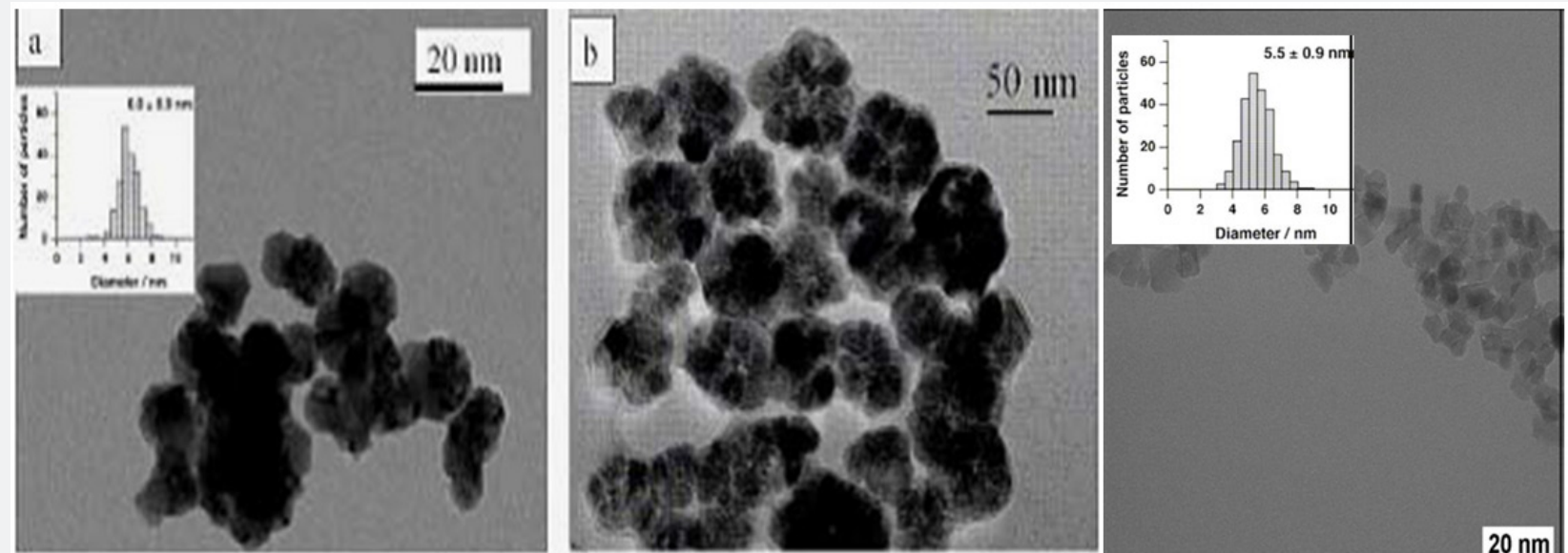

Figure 3: Cytotoxicity MTT Assay of Bacterial Nanoshells in Cancer Cell Lines and model animals.

TEM micrograph of the dispersed ZnGd-ferrite nanoparticles in ethanol and deposited over a $\mathrm{Cu}$ grid are shown in Figure 3 appearing almost spherical particles with a narrow size distribution. The histogram of the different diameters over 100 particles from a statistical analysis in the insert showed Gaussian distribution with mean \pm sd diameter $5.5 \pm 0.9 \mathrm{~nm}$ [37]. Both TEM 


\section{Cancer Therapy \& Oncology International Journal}

micrograph and XRD measurements indicated a high crystalline degree of all nanoshells [38]. Comments: Nanoshells are multicomponent systems. Each component is in specific physical phase. In different phases, components give one XRD peak but different TEM histograph. So, TEM and XRD give partly define the electronic densities matched with crystalline structure.

Moreover, these bacterial nanoshell systems generate sufficient heat (specific absorption rate SAR[W/g]) gradually and stop heating at Curie temperature Tc. During this process, power dissipated from the nanoshell systems over tumors can be calculated from area under hysteresis loop. Standard MTT protocols are available to test cytotoxicity (http://ctep.info. nih.gov) using synthesized nanoshells incubated with cancer cell lines. In earlier study, nanoshells showed no cytotoxicity by MTT assay on normal human and cancerous cell lines. No signs of toxicity were observed on $25 \mathrm{BalB} / \mathrm{c}$ mice 36 . Critical analysis: MTT method is gold standard to test cytotoxicity or tumor killing.

Basis of Magnetic Nanoparticle Theranostic Monitoring: Dependence of Magnetic Moments and Temperature

Nanoshells may serve as new MRI contrast agents for hyperthermia monitoring. They were prepared in viscous cellulose solutions to maintain them in suspension. The concentrations were $\mathrm{C}=0.29,0.57,1.14,2.28$, and $03.40 \mathrm{mmol} / \mathrm{kg}$ weight in addition to the control (i.e. $\mathrm{C}=0.0$ ) [33]. Authors established that relaxation rates were temperature-sensitive to anticipate that these nanoshells can be used as thermometry agents to improve the tumoricidal clinical procedures, e.g. hyperthermia and/or chemotherapy treatment. MR longitudinal (T1) and transverse (T2) relaxation enhancement vary and depend on dense tumor protons, hysteresis effects (magnetic moments) of thermosensitive nanoparticle concentrations of the $\mathrm{Zn}-\mathrm{Gd}-\mathrm{Fe}$ family in coated MNPs [33]

Critical analysis: Hysteresis effect on metals generates heat. Due to overheating by magnetic effect, nanometals change their relaxivities. So, relaxivities can monitor the hyperthermia effect of nanoparticles as well as tumor killing.

\section{Nanoshell Assisted MR Imaging of Tumor}

At $23^{\circ} \mathrm{C}$, Nanoshells showed specific behavior. The relaxation signal intensities vs TE for various gadolinium concentrations are shown in Figure 4. Single exponential curve was fitted to the data using non-linear programming (Levenberg-Marquardt) minimizing an error function to yield the relaxation time constant T2. R2 enhancement is shown as linear with $\mathrm{C}$ in Figure 5. The relaxivity ( $\mathrm{r} 2$ ) is defined by the equation ( $\mathrm{R} 2 \mathrm{I}=\mathrm{R} 20+\mathrm{r} 2 \mathrm{C}$ ) was 240 (s mmol/kg)-1. R20 is the transverse relaxation rate in the absence of MNPs. Similar analysis for the T1 data revealed $\mathrm{r} 1=$ $1.8(\mathrm{~s} \mathrm{mmol} / \mathrm{kg})^{-1}$.
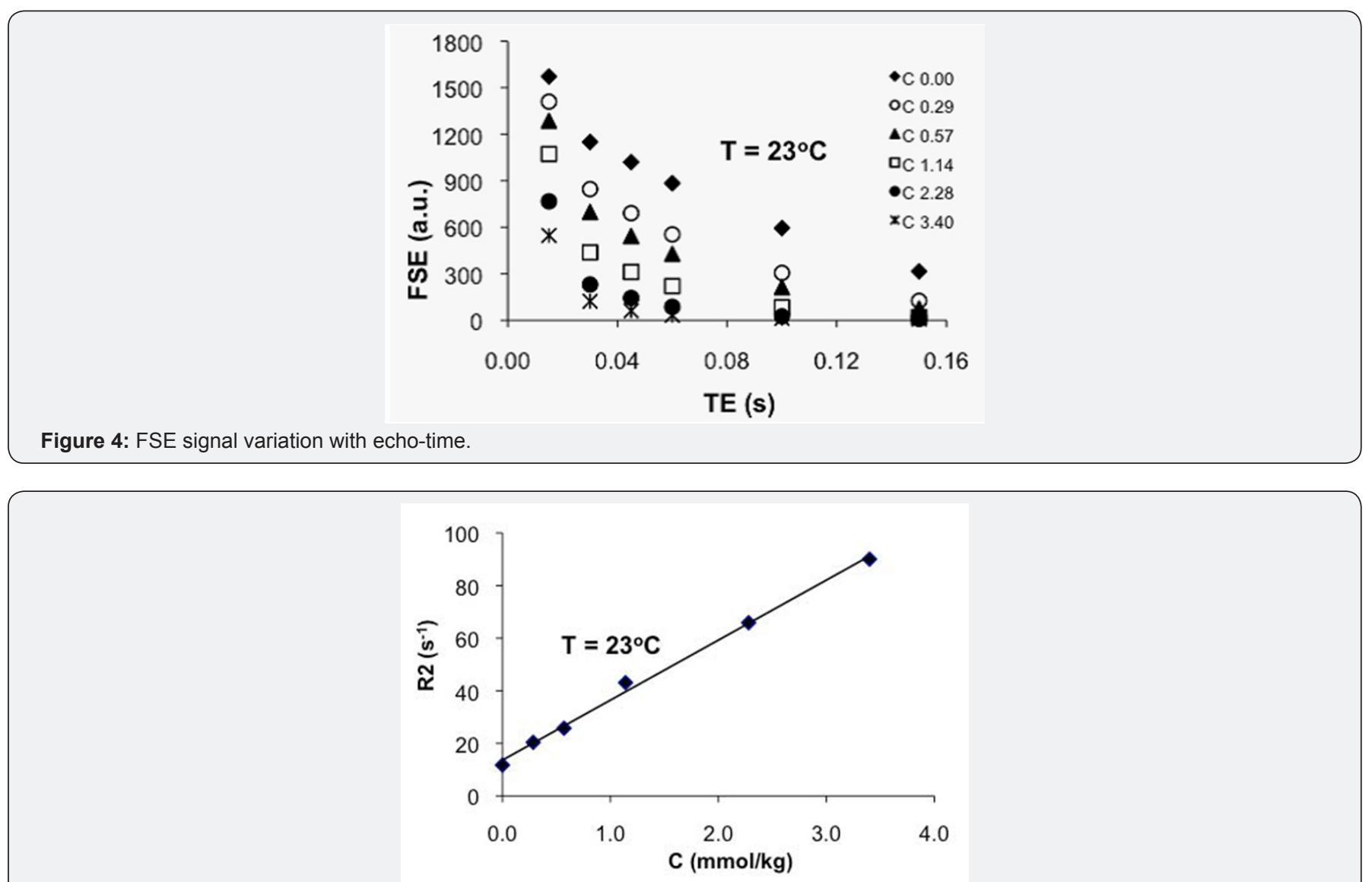

Figure 5: Transverse relaxation rate vs. concentration at temperature $=230 \mathrm{C}$. 


\section{Cancer Therapy \& Oncology International Journal}

Spin-echo T2 weighted images at different echo times (TE) showed signal decay variation with both concentrations (in different tubes) and TE is clearly demonstrated. The calculated values for the relaxation rates $\mathrm{R} 1$ and $\mathrm{R} 2$ are shown in Table 1 along with their ratio $\mathrm{R} 2 / \mathrm{R} 1$ at $23^{\circ} \mathrm{C}$. As expected for superparamagnetic particles R2 >> R1. The effect of MNPs will be to produce negative image contrast (i.e. signal reduction due to $\mathrm{T} 2$ wieghting is more pronounced than signal elevation due to T1 weighting) (Table 1).

Table 1: R1 and R2 values at different concentrations of the nanoparticles at 23oC.

\begin{tabular}{|c|c|c|c|}
\hline Nanoparticle concentration & R1(sec-1) & R2(sec-1) & R2/R1 \\
\hline 0 & 0.64 & 11.7 & 18.3 \\
\hline 0.025 & 0.6 & 20.4 & 29.9 \\
\hline 0.05 & 0.72 & 26.8 & 36.0 \\
\hline 0.1 & 0.80 & 43.1 & 52.4 \\
\hline
\end{tabular}

Upon heating the samples to temperatures blow and above the particles Curie temperature $\left(42.8^{\circ} \mathrm{C}\right)$ the $\mathrm{R} 1$ and $\mathrm{R} 2$ are measured for the different concentrations. Normalized values of R1 and R2 are plotted in Figures 6 and 7 respectively where it is shown that both relaxation rates increase linearly with both concentration of the MNPs and temperature. Critical analysis: Temperature affects the proton spinning rate in superparamagnetic particles. Near
Curie temperature, it tumbles faster. So, relaxivities also changes depending upon TE (echo time) in inversely proportion. At Curie temperature and low TE, relaxivities indicate the distorted tumor tissue protons as ideal indicator of physical state of nanoshells fully releasing nanoparticles to show tumor cytotoxicity. It may be optimal therapeutic condition in cancer intervention.

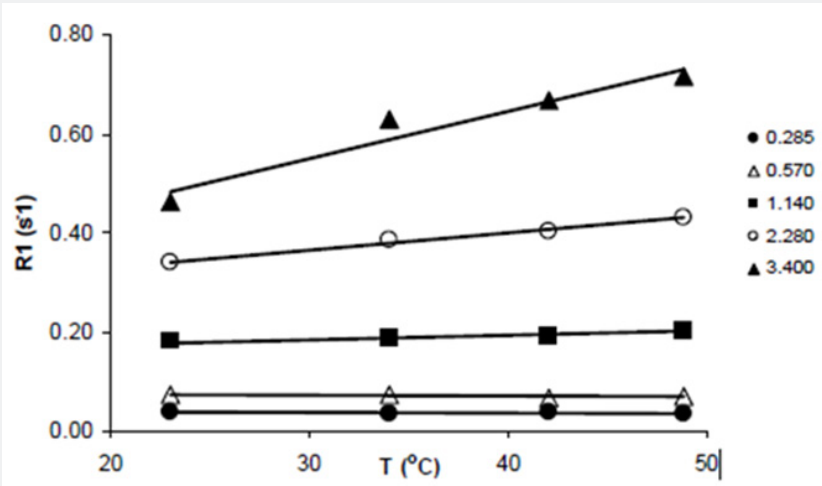

Figure 6: The linear variation of 1/T1 (normalized) with temperature increases with the concentration of MNPs.

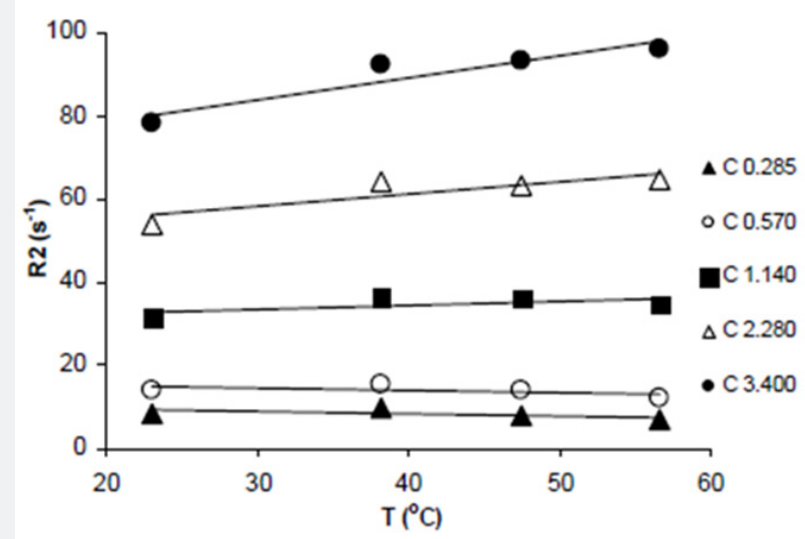

Figure 7: As for 1/T1 the variation of 1/T2(normalized) is linear with temperature, however, the slopes are larger.

The relaxivity ( $\mathrm{r} 2$ ) values (in $\mathrm{kg} \mathrm{s}-1 \mathrm{mmol}-1$ ) are summarized in Table 2. Relaxivity increases linearly with temperature and data has a positive slope 0.173 and $\mathrm{R}$-squared value 0.92 . MR relaxation enhancement is demonstrated with new class of magnetic nanoparticles that have already been used for hyperthermia applications. The contrast ability (in particular R2) is found to 
vary linearly and significantly with concentration. Furthermore, the relaxation enhancement of these new contrast agents exhibits linear variation with temperature. This would allow the use of these MNPs particles, in addition to their role as contrast agents, in temperature monitoring and hyperthermia applications. Critical analysis: For structural details of tumor size and shape, R1 relaxivities are significant while R2 relaxivities signify tumor tissue contrast.

\section{Bacterial Drug Delivery System for Hyperthermia}

The use of non-pathogenic bacteria in drug delivery for cancer therapy is an innovative approach. Salmonella typhimurium strains accumulate at tumor sites when injected in tumor-bearing mice [39-44]. These bacteria are ideal carriers for contrast agents, for gene vectors [45], vaccines [46] and chemotherapeutic agents $[47,48]$. However, loading bacteria with MNPs is difficult. Bacterial delivery system transport cancer drugs to the tumor site. Salmonella BR 509 was used to encage MNPs to transport at tumor site. These bacteria are rod shaped with 1 micron in length. Ultrafine 1 micron rod shaped particles inside Salmonella are shown in Figure 8 by SEM micrography of the MNP-bacteria. It was observed that small size particles were diffused into the cell membrane.



a

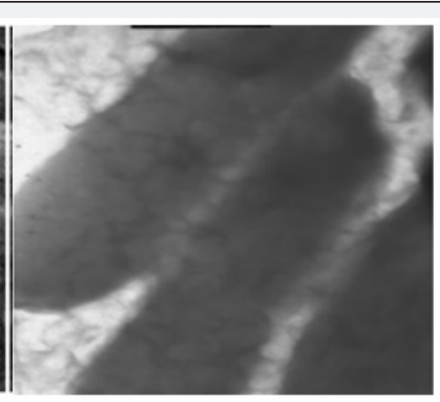

b



C

Figure 8: a. SEM of encapsulated MNPs attached on the cell membrane of Salmonella,

b. Smaller NMPs entered the Salmonella's membrane and

c. Vertically aligned Salmonella when exposed to a magnetic field placed on top of the slides.

Strains of attenuated Salmonella typhimurium BRD509 encage the drug-coated MNPs. These loaded Salmonella organisms administer anti-cancer drug to tumor without any toxicity of high doses of chemotherapeutic drugs. Anticancer drug (5FU or doxirobucine) in a thermosensitive Salmonella shell carried the coated shells to tumor tissue. Under the influence of a magnetic field at $0.963 \mathrm{MHz}$, the tumor-targeted MNP inside Salmonella shell generated heat up to $42^{\circ}$ to $43^{\circ} \mathrm{C}$, and releasing the drug at the tumor site [45].

Critical Comments: Avirulent Salmonella organism host MNPs at optimal temperatures. However, in living animals, macrophages engulf bacteria faster. So, it is necessary to keep phagocytic activity minimal. Delivery mode of nanoparticle loaded bacteria is very important. Injection by ip or through tail is not ideal method. Intravenous route is preferred with real-time monitoring of tumor size.

Figure 8 illustrates the loss of non-MNP-associated Salmonella organisms, following co-incubation with MNPs at $24^{\circ} \mathrm{C}$ for 120 minutes, after each cycle of wash. Figure 9 shows the temperature distribution immediately following non-invasive magnetic heating of shells carrying drug load and MNPs and injected ip into the tail of the treated mouse. Close up pictures were taken for tumor to present significant difference. It was noticed that blood flow to tumor was increased due to increase in temperature. The survival measurements showed that hyperthermia treated mice with encapsulated drug have survived the longest ( 5 times more than drug alone treated mice) a subsequent measurement of controlled tumor growth (Figure 10). 


\section{Cancer Therapy \& Oncology International Journal}

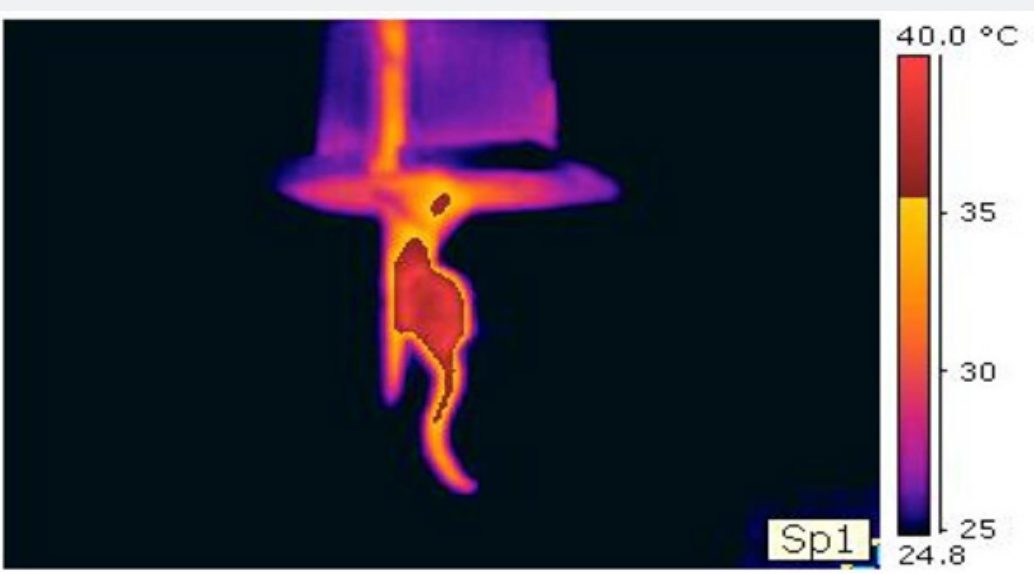

Figure 9: Temperature map of heated tumor containing MNPs. The inner pictures show the tumor just before introducing the treatment.

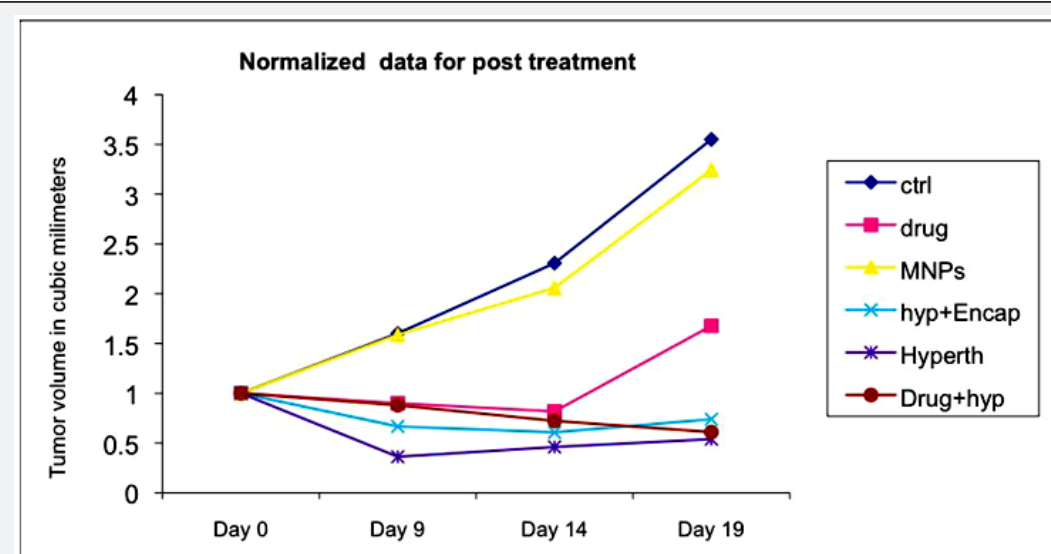

Figure 10: Tumor volumes for all groups. The tumor volume is normalized with the control starting the first day of treatment. Day 0 is day when treatment started and the 30th day after the inoculations of the tumor.

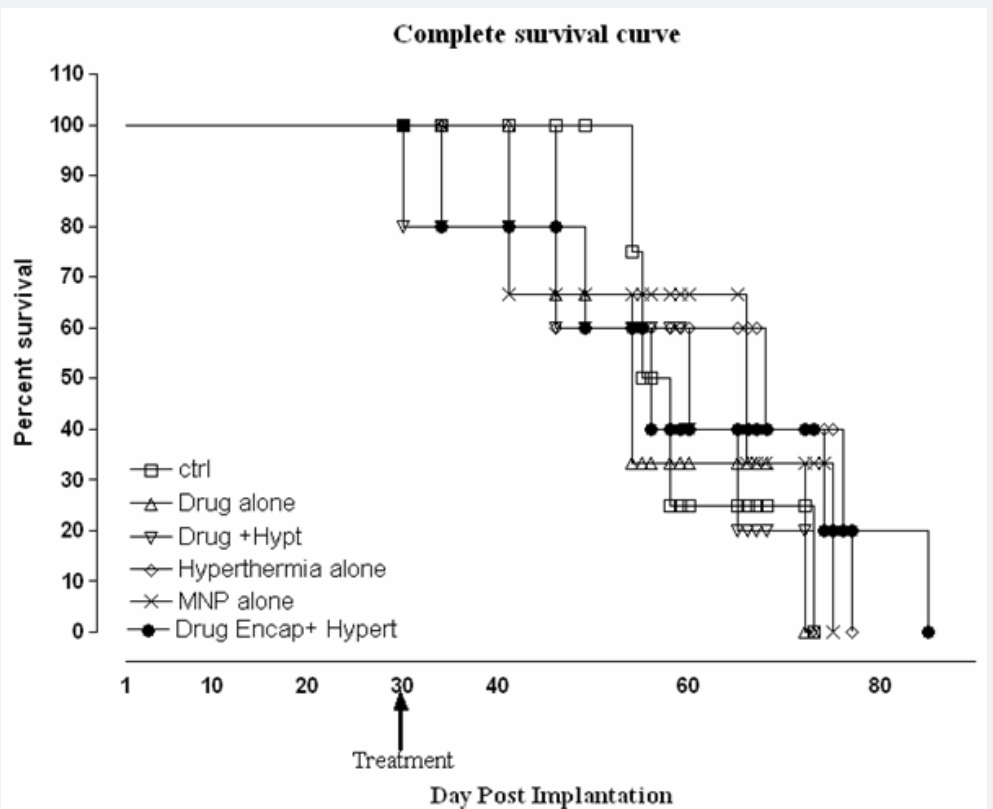

Figure 11: Tumor volumes for all groups. The tumor volume is normalized with the control starting the first day of treatment. Day 0 is day when treatment started and the 30 th day after the inoculations of the tumor. 


\section{Cancer Therapy \& Oncology International Journal}

It is clearly shown that the multifunctional therapy has better efficacy than each of the therapies if they administered alone. Figure 11 shows the survival curve for all groups. It is clear that the volume of the tumor has reduced dramatically and the animals survived the most when the hyperthermia therapy was implemented.

Comments: In different post-treatment days, effect of MNP induced tumor temperature rise should not be considered as interventional hyperthermia to show reduction in tumor size. Tumor vascularity, permeability and extravascular space should be measured easily by dynamic contrast enhanced data for tumor response to therapy or prevention from cancer or tumor growth further. However, hyperthermia with controlled released MNP may enhance animal survival.

Figures 12 \& 13 demonstrates that when hyperthermia is utilized the effectiveness of delivery and treatment are better. Bacterial delivery of MNPs is effective. The mechanism of drug unloading or controlled release of MNPs is not fully understood, but may be associated with the bacteria cell membrane conformational changes inside the tumor dividing cells.

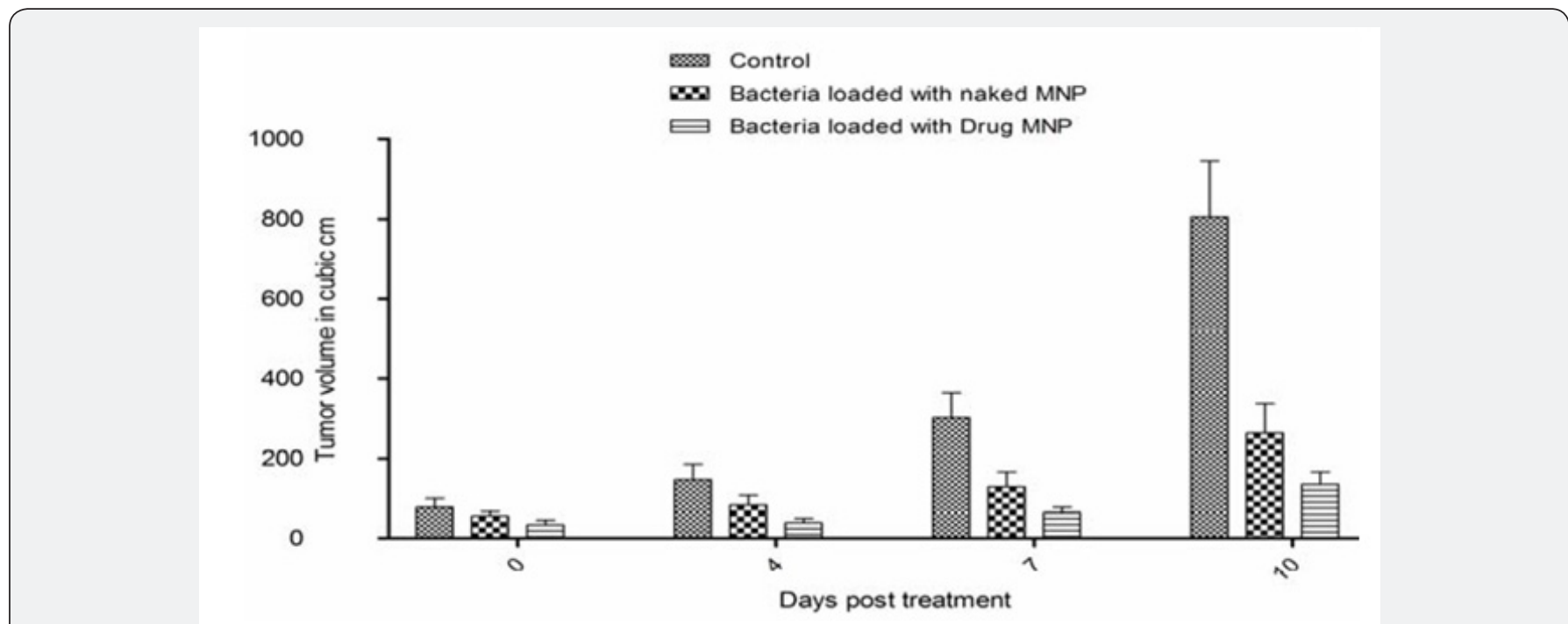

Figure 12: Tumor volume post treatment of control, bacterial loaded with MNPs (no drug) and bacteria loaded with shelled drug and MNP.



a.

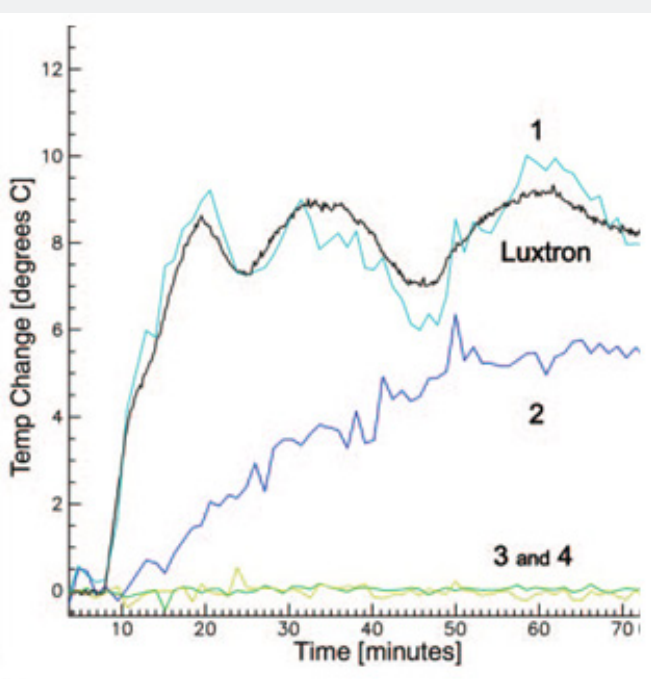

b.

Figure $13(\mathbf{a}, \mathbf{b})$ : Estimates of temperature change (starting at body temperature) during hyperthermia therapy in a patient with a soft-tissue sarcoma of the lower leg. (a) Axial image of the calf and the tumor shows the anatomy. The leg is surrounded by a water bolus and the heating antennae are on the outside of the cylinder, as shown in Figure 1. (b) Graph shows the temperature changes during the 70 minutes of therapy. The different lines correspond to the ROls, which are shown as color blocks in c and d. 1 ROI in the tumor near the invasive fiberoptic temperature probe (black line on graph), $2_{\text {_ }} \mathrm{ROI}$ in normal muscle, 3 and 4 _ ROIs in the oil references. The drift correction has been applied to the graph, as demonstrated by the near-constant values of the oil references. 


\section{Cancer Therapy \& Oncology International Journal}

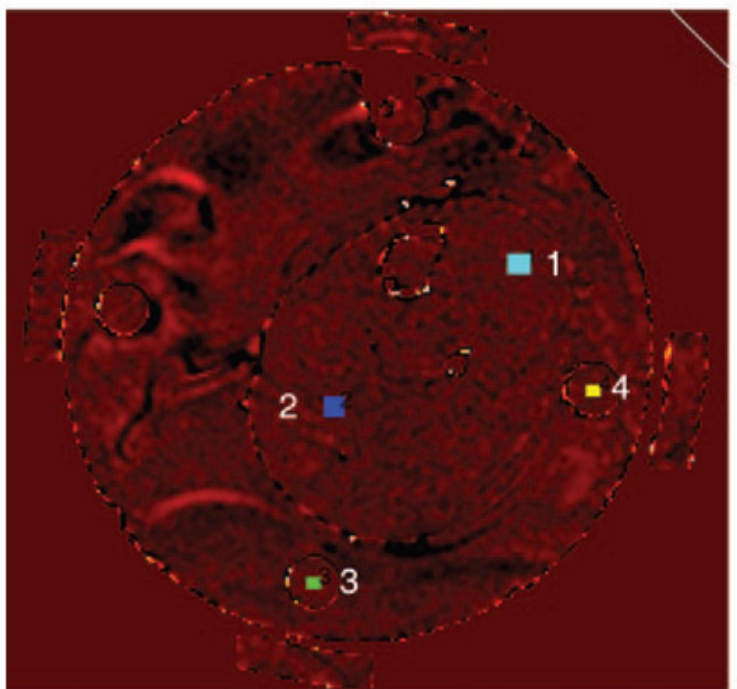

c.

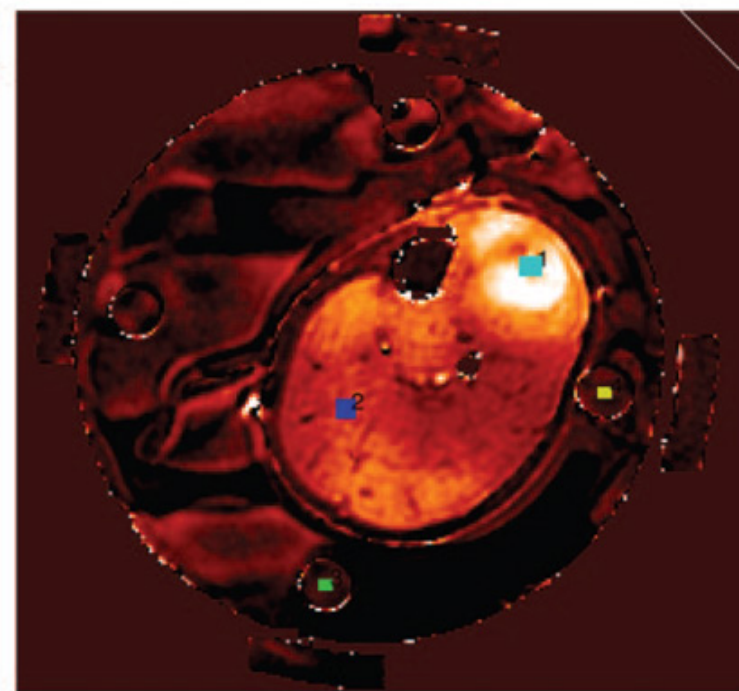

d.

Figure 13 (c, d): Anatomy images obtained at the beginning (c) and near the end (d) of therapy show the regional temperature changes by means of an overlaid orange-toned temperature scale.

\section{What is Accomplished?}

Diagnosis and therapeutic monitoring of malignant tissues such as neoplasia, fibroma, sarcoma can be done utilizing contrast nanoshells and heating magnetic nanoparticles delivered to tumor sites encaged inside attenuated strains of bacteria target at tumor sites in order to prevent cancer. Real-time imaging is ideal tool in theranostic monitoring (Figure 14).

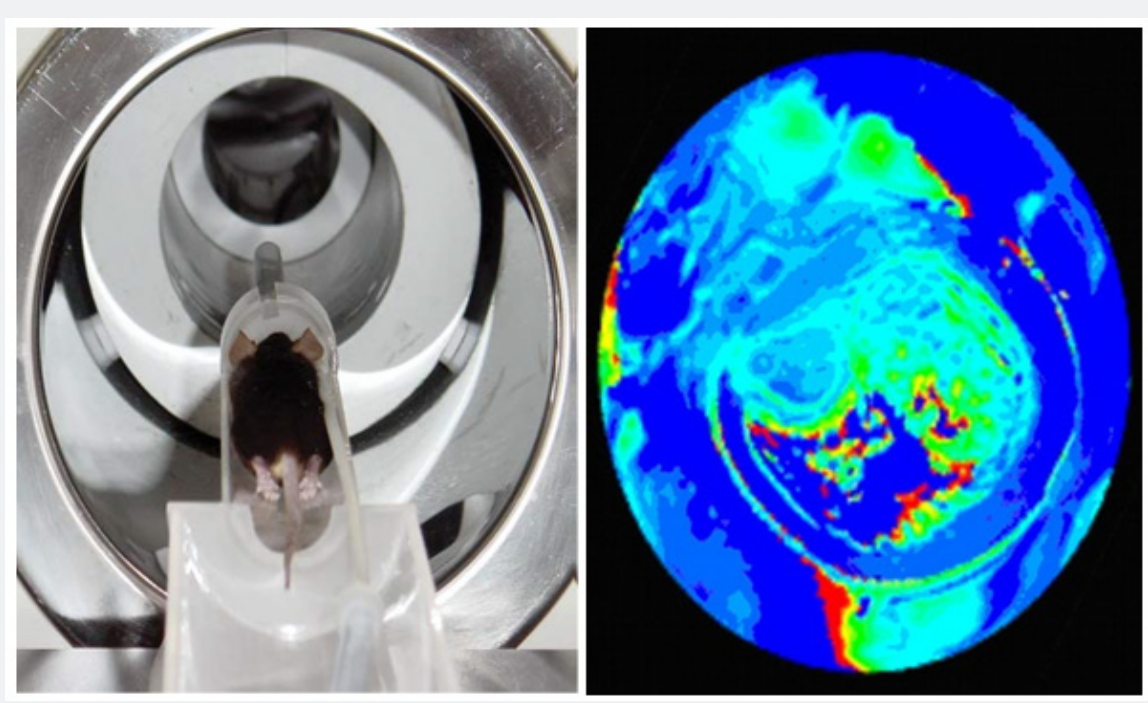

Figure 14: Molecular imaging instrument and theranostic nanoparticles.

Present State of Art on Theranostic monitoring of Tumor and Nanodrugs

Theranostic monitoring mechanisms depend on drug activation, targeted molecule accumulation, and enhancement of contrast in images. Theranostic signals may arise from targeting molecules associated with cell-surface receptors internalizing into cytosol. New trend is developed to design nanoparticles made from proteins, peptides, polymers, lipids, metals, metal oxides and carbon nanotubes coating drug conjugates, complexes, dendrimers, vesicles, micelles, microbubbles functionalized for therapeutic drug carriers and imaging contrast or theranosis. Author reviewed temperature sensitive phase-contrast MRI method to predict tumor recovery using apparent diffusion coefficient technique and suggested the feasibility: 
i. Hyperthermia can be an effective adjunctive therapy, but adequate attention must be paid to ensure the target temperatures are achieved for an adequate period of time.

ii. MR imaging can monitor temperature throughout a volume and obtains useful morphometric and functional information from tumor and normal tissues that makes it the modality of choice to image regional hyperthermia therapy delivery.

iii. The bond length between the hydrogen and the oxygen in the water becomes longer with temperature increases; thus, the chemical shielding of the proton is reduced or increased chemical shift.

iv. Use of reference nanomaterials also measures absolute temperature as function of fat-water frequency difference as function of absolute temperature, due to mixtures of fat and water such as breast cancer tissue.

v. Heatable tumor tissues are best in conducting successful clinical trials of hyperthermia.

\section{Conclusion}

In conclusion, functionalization of a potential imaging guided intervention of multifunctional magnetic nanoparticles provides targeted drug delivery and non-invasive monitoring of drug localization by thermometry. A smart drug carrier with high affinity to tumors can be utilized to site-specific delivery of thermosensitive shells bearing anticancer drug and nanoparticles. Non-invasive localized heating can be induced by a remote alternating magnetic field. The intervention when applied to induced tumors may be preferred therapeutic management of the tumor.

\section{Appendix 1}

\section{Bacterial Nanoshell Assisted MR Imaging}

At 1.5 T field strength, Inversion Recovery and Fast Spin-Echo imaging sequences were used to image animal tumor size with the following parameters: a. Inversion Recovery pulse sequence for the $\mathrm{T} 1$ measurement with $\mathrm{TI}=50,100,300,700,1200,2000$, and 3000 ms; with a TE = 15 ms; Spin-echo pulse sequence for the T2 measurement with $\mathrm{TE}=15,30,45,60,100$, and $150 \mathrm{~ms}$ [33].

In these experiments, 1 x 108 colony forming units (CFUs) of Salmonella strain BRD509 were incubated with MNPs in saline buffer. At the end of the incubation period, the bacterial suspension was spun down and the supernatant was aspirated. After resuspending the bacterial pellet in $1 \mathrm{ml}$ saline, the bacterial suspension was subjected to a 0.45 Tesla permanent magnet for 15 minutes on the outside surface of the eppendorf tube. The remaining supernatant, presumably containing non-MNPassociated bacteria, was aspirated, and replaced with fresh saline. This procedure was repeated 3 times in total. Aliquots were removed from the bacterial suspension before and after each wash cycle and plated to determine the actual count of bacterial CFUs.
Using this procedure, the number of bacterial CFUs remaining after four cycles of magnetic separation and washing (which most likely represents the number of bacteria actually associated with MNPs) was determined, and hence the \% of bacteria associated with MNPs was calculated. It demonstrates that all bacteria not associated with nanoparticles are effectively removed by the 3rd wash cycle. Furthermore, varying the incubation conditions has a clear impact on the uptake of MNPs by the bacteria.

Incubation of MNPs with live Salmonella organisms at room temperature resulted in the most optimal uptake, which was about $6 \%\left(6 \times 10^{6}\right.$, sufficient for the loading purpose). The fact that the association appears to be strong suggests that it is feasible to use the MNP-loaded, or MNP associated, Salmonella organisms in tumor targeting in vivo. B6 mice $(20 \mathrm{~g} \pm 3 \mathrm{~g})$ were housed in appropriate isolated caging with sterile rodent laboratory chow and acidified water ad libitum and a 12-h light/dark cycle. B6 mice were implanted subcutaneous in the mid of tail by 50 micro liter volume of PBS with approx. 300,000 melanoma cells, 99\% viability. Tumor was visible after 20 days, $1-2 \mathrm{~mm}$ diameter. Four groups of five each were prepared such that one group with no treatment (control), second group with drug therapy (5FU) only, third group with hyperthermia only and the fourth group with integrated particle that has the drug and heating agent encapsulated in the thermosensitive polymer. The treatment started after a month from inoculation of the cells.

In this experiment control group was given saline, hyperthermia (nanoparticles alone) group received nanoparticles in a concentration of $20 \mathrm{mg} / \mathrm{kg}$ of body weight and followed by hyperthermia at $0.963 \mathrm{MHz}$ for 45 minutes. Six doses were given after every two days gap. Tumor volume was measured every two days until survival of mice. Tumor volume corresponds to width ${ }^{2}$ $\mathrm{X}$ length / 2. Tumor temperature rises till $42-43^{\circ} \mathrm{C}$, and was monitored during the experiment [33].

\section{References}

1. Shinkai M (2000) Functional magnetic particles for medical application. J Biosci Bioeng 94(6): 606-613.

2. Mitsumori M, Hiraoka M, Shibata T, Okuno Y, Nagata Y, et al. (1996) Targeted hyperthermia using dextran magnetite complex: a new treatment modality for liver tumors. Hepatogastroenterology, 43(12): 1431-1437.

3. Minamimura T, Sato H, Kasaoka S, Saito T, Ishizawa S, et al. (2000) Tumor regression by inductive hyperthermia combined with hepatic embolization using dextran magnetite-incorporated microspheres in rats. Int J Oncol 16: 1153-1158.

4. Hilger I, Fruhauf K, Andra W, Hiergeist R, Hergt R, et al. (2002) Acad Radiol 9: 198-202.

5. Moroz P, Jones SK, Gray BN (2002) Magnetically mediated hyperthermia: current status and future directions. Int $\mathrm{J}$ Hyperthermia 18(4): 267-284.

6. Cavalier R, Ciocatto EC, Giovanel B, Heidelbe C, Johnson, et al. (1967) Cancer 20(9): 1351-1381.

7. Stauffer PR, Cetas TC, Fletcher AM, Deyoung DW, Dewhirst MW, et al. (1984) Observations on the use of ferromagnetic implants for inducing hyperthermia. IEEE Trans Biomed Eng 31(1): 76-90. 


\section{Cancer Therapy \& Oncology International Journal}

8. Lin JC, Wang YJ (1987) Interstitial microwave antennas for thermal therapy. Int J Hyperthermia 3(1): 37-47.

9. Ikeda N, Hayashida O, Kameda H, Ito H, Matsuda T (1994) Experimental study on thermal damage to dog normal brain. Int J Hyperthermia 10(4): 553-561.

10. Wust P, Hildebrandt B, Sreenivasa G, Rau B, Gellermann J, et al. (2002) Hyperthermia in combined treatment of cancer. Lancet Oncol 3(8): 487-497.

11. Van der Zee J (2001) Heating the patient: a promising approach? Ann Oncol 13(8): 1173-1184.

12. Moroz P, Jones S K, Gray BN (2001) Status of hyperthermia in the treatment of advanced liver cancer. J Surg Oncol 77(4): 259-269.

13. Jordan A, Scholz R, Maier-Hauff K, Johannsen M, Wust P, et al. (2001) Magnet Magn Mater 225(1-2): 118-126.

14. Gilchrist RK, Medal R, Shorey WD, Hanselman RC, Parrott JC, et al. (1957) Selective inductive heating of lymph nodes. Ann Surg 146(4): 596-606.

15. Mosso JA, Rand RW (1972) Ferromagnetic silicone vascular occlusion: a technic for selective infarction of tumors and organs. Ann Surg 178(5): 663-668.

16. Rand RW, Snyder M, Elliott DG, Snow HD (1976) Bull. Los Angeles Neurol Soc 41: 154-159.

17. Gordon RT, Hines JR, Gordon D (1979) Intracellular hyperthermia. A biophysical approach to cancer treatment via intracellular temperature and biophysical alterations. Med Hypotheses 5(1): 83-102.

18. Rand RW, Snow HD, Elliott DG, Snyder M (1981) Thermomagnetic Surgery for cancer. Appl Biochem Biotechnol 6(4): 265-272.

19. Borrelli NF, Luderer AA, Panzarino JN (1984) Hysteresis heating for the treatment of tumours. Phys Med Biol 29(5): 487-494.

20. Hase M, Sako M, Hirota S (1990) Nippon-Igaku-Hoshasen-GakkaiZasshi. 50: 1402-1414.

21. Suzuki S, Arai K, Koike T, Oguchi KJ (1990) Studies on liposomal ferromagnetic particles and a technique of high frequency inductive heating- in vivo studies of rabbit. Japan Soc Cancer Therapy 25: 26492658.

22. Chan DCF, Kirpotin DB, Bunn PA Jr (1993) Synthesis and evaluation of colloidal magnetic iron oxides for the site-specific radiofrequencyinduced hyperthermia of cancer. J Magn Magn Mater 122(1-3): 374378.

23. Jordan A, Scholz R, Wust P, Fahling H, Krause J, et al. (1997) Effects of magnetic fluid hyperthermia (MFH) on $\mathrm{C} 3 \mathrm{H}$ mammary carcinoma in vivo. Int J Hyperthermia 13(6): 587-605.

24. Jordan A, Scholz R, Wust P, Fahling H, Felix R (1999) Magnetic fluid hyperthermia (MFH): Cancer treatment with $\mathrm{AC}$ magnetic field induced excitation of biocompatible super paramagnetic nanoparticles

J Magn Magn Mater 201: 413-419.

25. Stauffer PR, Cetas TC, Fletcher AM, DeYoung DW, Dewhirst MW, et al. (1984) Observations on the use of ferromagnetic implants for inducing hyperthermia. IEEE Trans Biomed Eng 31(1): 76-90.

26. Xu XJ, Chew CH, Siow KS, Wong MK, Gan LM (1999) Langmuir 15: 8067.
27. Hilger I, Fruhauf K, Andra W, Hiergeist R, Hergt R, et al. (2002) Heating potential of Iron Oxides for therapeutic purposes in interventional radiology. Acad Radiol 9(2): 198-202.

28. Haik Y, Chen CJ (2005) US Patent Application.

29. Martin Bettge, Jhunu Chatterjee, Yousef Haik (2004) Physically synthesized Ni-Cu nanoparticles for magnetic hyperthermia. J Biomag Res Tech 2: 4 .

30. Chatterjee J, Bettge M, Haik Y, Chen CJ (2005) Synthesis and characterization of polymer encapsulated $\mathrm{Cu}-\mathrm{Ni}$ magnetic nanoparticles for hyperthermia applications. J Mag Mag Mat 293: 303309.

31. Sharma R, Haik Y, Chen CJ (2007) Super paramagnetic iron oxide-myoglobin as potential nanoparticle: iron oxide myoglobin binding properties and magnetic resonance imaging marker in mouse imaging. J Exper Nanoscience 2(1): 127-138.

32. Ishihara Y, Calderon A, Watanabe H, Okamoto K, Suzuki Y, et al. (1995) A precise and fast temperature mapping using water proton chemical shift. Magn Reson Med 34(6): 814-823.

33. Parker DL (1983) Applications of NMR imaging in hyperthermia: an evaluation of the potential for localized tissue heating and non invasive temperature monitoring. IEEE Trans Biomed Eng 31(1): 161-167.

34. Le Bihan D, Delannoy J, Levin RL (1989) Temperature mapping with MR imaging of molecular diffusion: application to hyperthermia. Radiology 171(3): 853-857.

35. Rieke V, Pauly KB (2008) MR thermometry. J Magn Reson Imaging 27(2): 376-390

36. Chen CJ, Haik Y, Chatterjee J (2003) Recent Research Developments in Magnetism and Magnetic Materials, Tranworld Research Network, India.

37. Chen CJ, Hayek S, Mohite V, Yuan H, Chatterjee J, et al. (2006) In Cancer Nanotechnology-Nanomaterials for Cancer Diagnosis and Therapy. Nalwa HS and Webster T (Eds), Chapter 11, pp. 500.

38. Jain RK, Forbes NS (2001) Can engineered bacteria help control cancer? Proc Natl Acad Sci USA 98(26): 14748-14750.

39. Al Ramadi BK (2002) Mol Immunol 38: 931-940.

40. Al Ramadi BK (2003) Mol Immunol 39: 763-770.

41. Al Ramadi BK (2004) Mol Immunol 40: 671-679.

42. Al Ramadi BK (2004) Microbes Infect 6: 350-359.

43. Clairmont C, Lee KC, Pike J, Ittensohn M, Low KB, et al. (2000) Biodistribution and genetic stability of the novel antitumor agent VNP20009, a genetically modified strain of Salmonella typhimurium. J Infec Dis 181(6): 1996-2002.

44. Bermudes D, Zheng LM, King IC 5(2): 194-1999.

45. Jia LJ, Wei DP, Sun QM, Jin GH, Li SF, et al. (2007) Inter J Cancer.

46. Pawelek JM, Low KB, Dermudes D (1997) Cancer Res 20: 4537-4544.

47. Reisfeld RA, Niethammer AG, Luo Y, Xiang R (2004) DNA vaccines suppress tumor growth and metastases by the induction of antiangiogenesis. Immun Rev 199: 181-190.

48. MacFall, JR, Sohar BJ (2007) Radiographics 27: 1809-1818. 
Your next submission with Juniper Publishers will reach you the below assets

- Quality Editorial service

- Swift Peer Review

- Reprints availability

- E-prints Service

- Manuscript Podcast for convenient understanding

- Global attainment for your research

- Manuscript accessibility in different formats ( Pdf, E-pub, Full Text, Audio)

- Unceasing customer service

Track the below URL for one-step submission https://juniperpublishers.com/online-submission.php 